\title{
Cannabis use during methadone maintenance treatment for opioid use disorder: a systematic review and meta-analysis
}

\author{
Heather McBrien, Candice Luo BHSc, Nitika Sanger BHSc, Laura Zielinski MSc, Meha Bhatt MSc, \\ Xi Ming Zhu MBBS, David C. Marsh MD, Lehana Thabane PhD MSc, Zainab Samaan MBChB PhD
}

Abstract

Background: Rates of cannabis use among patients receiving methadone maintenance therapy are high, and cannabis use may be associated with outcomes of methadone maintenance therapy. We examined the effect of cannabis use on opioid use in patients receiving methadone maintenance therapy to test the hypothesis that cannabis use is associated with a reduction in opioid use.

Methods: In this systematic review, we searched MEDLINE/PubMed, Embase, PsycINFO, CINAHL and ProQuest Dissertations and Theses Global from inception to July 12,2018 . We summarized the effects of cannabis use on opioid use during methadone maintenance therapy and treatment retention. We conducted meta-analyses using a random effects model.

Results: We included 23 studies in our review. We performed a meta-analysis of 6 studies, with a total number of participants of 3676, examining use of cannabis and opioids during methadone maintenance therapy. Owing to high heterogeneity, we described the studies qualitatively but provide the forest plots as supplemental material. The overall quality of evidence was very low, with a high risk of bias, owing to the nature of observational studies.

Interpretation: We found no consensus among studies that cannabis use is associated with reduced opioid use or longer treatment retention when used during methadone maintenance therapy in patients with opioid use disorder. PROSPERO Registration: CRD42015029372

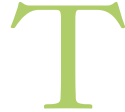
he opioid epidemic related to an increase in prescriptions $^{1-4}$ has escalated in the past 2 decades. Opioid use disorder is a fundamental component of this crisis. ${ }^{5,6}$ Despite the morbidity and mortality due to opioid use disorder, high rates of HIV infection and hepatitis $\mathrm{C}$ among patients with the disorder and high unemployment rates, treatment options are limited in scope and effectiveness. ${ }^{78}$ Methadone maintenance therapy is the most common treatment for opioid use disorder, ${ }^{9,10}$ although other treatments are available. ${ }^{11}$ Despite the reported benefits of methadone maintenance therapy in managing opioid use disorder, the number of patients receiving this therapy who continue to use illicit opioids is high. ${ }^{12}$ The rates of cannabis use among patients receiving methadone maintenance therapy are higher than those in the general population: about a third of Canadians have used cannabis once in their lifetime, ${ }^{13}$ whereas $59.7 \%$ of males and $43.5 \%$ of females receiving methadone maintenance therapy reported having used cannabis. ${ }^{14-16}$ Research suggests that polydrug use (aside from cannabis) is prevalent in the methadone maintenance therapy population. ${ }^{17,18}$ There is limited evidence to suggest that cannabis use may reduce opioid use in pain management ${ }^{19}$ but not in opioid use disorder. ${ }^{20}$ Studies have shown that, in US states with dispensary-based medical cannabis laws, fewer prescription opioids are dispensed, ${ }^{21}$ and there are fewer opioid-related deaths. ${ }^{22}$ The US Department of Veterans Affairs has suggested that cannabis should be legalized not only as a mechanism to lower prescription opioid use but also to manage opioid withdrawal. ${ }^{23}$ This report made headlines labelling cannabis as an "exit drug."

However, the "exit hypothesis" - the idea that cannabis can be used to manage withdrawal symptoms and therefore help patients with opioid use disorder to stop using opioids has not been examined systematically. With the legalization of

Competing interests: Laura Zielinski reports personal fees from Canadian Cannibis Clinics. No other competing interests were declared.

This article has been peer reviewed.

Correspondence to: Zainab Samaan, samaanz@mcmaster.ca CMAJ Open 2019. DOI:10.9778/cmajo.20190026 
cannabis in Canada, this question is relevant. We examined the relation between cannabis use and opioid use during methadone maintenance therapy. We focused our question on methadone maintenance therapy as it is the most commonly used treatment for opioid use disorder. We sought to determine 1) whether patients using cannabis during methadone maintenance therapy have lower rates of opioid use during therapy and 2) whether cannabis use improves treatment retention in opioid use disorder.

\section{Methods}

This review is presented according to Preferred Reporting Items for Systematic Reviews and Meta-Analyses (PRISMA) guidelines. ${ }^{24}$ It is registered with PROSPERO (no. CRD42015029372). The protocol with detailed methods was published elsewhere. ${ }^{15}$ The protocol included our target population, intervention, comparison, outcomes, search terms and a detailed search strategy (Supplemental Table S1, Appendix 1, available at www.cmajopen.ca/content/7/4/ E665/suppl/DC1). We provide a summary of the methods below.

\section{Information sources and study selection}

We searched MEDLINE/PubMed, Embase, PsycINFO, CINAHL and ProQuest Dissertations and Theses Global from inception to July 12, 2018 for relevant studies. We applied no language or demographic restrictions. We formulated our study question using the PICO (Patient, Intervention, Comparison, Outcome) model: In patients with opioid use disorder receiving methadone maintenance treatment, is cannabis use associated with illicit opioid use, treatment retention, polydrug use, criminal activity or risk behaviours for HIV infection (injection drug use, needle sharing and unprotected sex)? We included both studies with observational designs and those with randomized controlled trial designs.

\section{Data collection}

Included studies looked at the association between cannabis use and outcomes of methadone maintenance therapy. To be included, a study had to measure methadone maintenance therapy outcomes by reporting participants' continued opioid use during treatment or treatment retention rates by cannabis use. We excluded studies in which other treatments for opioid use disorder such as buprenorphine were used. We included only methadone maintenance therapy because it was the most commonly used treatment and to avoid the heterogeneity that would have resulted from including different treatment interventions. All articles were screened independently in duplicate at all stages, including data extraction (H.M., C.L., L.Z., M.B. and X.M.Z.). We measured interrater agreement with the $\kappa$ statistic. Risk of bias was assessed independently in duplicate (H.M., C.L., L.Z., M.B. and X.M.Z.) with the use of the modified NewcastleOttawa Scale, as all our included studies were observational by design. ${ }^{25}$ We measured the overall quality of the evidence using the Grading of Recommendations Assessment, Development and Evaluation (GRADE) framework. ${ }^{26}$

\section{Statistical analysis}

As per our protocol, the intent was to meta-analyze the primary studies and provide quantitative summary estimates. However, when we performed meta-analyses, using random effects taking into account heterogeneity expected in observational studies, the high heterogeneity precluded meaningful conclusions, and therefore we provide a description of the included studies and their findings qualitatively. We determined heterogeneity using the $I^{2}$ statistic; a value greater than $40 \%$ indicated high heterogeneity. ${ }^{27}$

We used RevMan version 5.3 software (Cochrane Community) for the meta-analyses. ${ }^{28}$ Some studies measured cannabis use both before and during treatment, and we chose to use the in-treatment cannabis measurement, as we were interested in how current cannabis use affects outcomes of current methadone maintenance therapy. In studies that included multiple follow-up time points for the outcome measurement, we included the latest follow-up point in the meta-analysis. We did not assess publication bias, as it has been shown that funnel plots do not accurately depict publication bias for meta-analyses that have fewer than 10 studies. ${ }^{29}$ However, we provide funnel plots of publication bias for illicit opioid use and treatment retention in Supplemental Figures S1 and S2, respectively, Appendix 1.

We performed a sensitivity analysis by excluding all studies with Newcastle-Ottawa Scale scores of 0 or 1 on individual questions. We performed further subgroup by country and method of cannabis use measurement (subjective v. objective) to further explain any significant heterogeneity found. We were unable to perform subgroup analyses by length of follow-up owing to the limited number of studies.

\section{Ethics approval}

As this study was solely literature based, it was not eligible for institutional ethics approval, and none was sought.

\section{Results}

Among the 2467 unique citations screened, 23 studies were included in the qualitative synthesis (Figure 1). ${ }^{13,14,17,20,30-48}$ Interrater agreement was acceptable for both title/abstract $(\kappa=0.63,95 \%$ confidence interval $0.57-0.69)$ and full-text screening $(\kappa=0.60,95 \%$ confidence interval $0.45-0.74)$. Although we did not apply any age restrictions, all studies were of adult populations. In studies that reported the proportion of participants with any recent or current (i.e., not lifetime measurements) cannabis use, the prevalence varied from $11.2 \%$ to $78.6 \% .{ }^{35,41}$ As the meta-analyses had high heterogeneity, we present forest plots of illicit opioid use during treatment by cannabis use and treatment retention in Supplemental Figures S3 and S4, respectively, Appendix 1.

Of the 23 studies, 12 looked at continued opioid use, ${ }^{12-14,30-38}$ 11 examined treatment retention in relation to using cannabis during methadone maintenance therapy, ${ }^{13,17,31,32,34,39-43,46}$ 
10 looked at polydrug use, ${ }^{13,30,31,35,37,43-47} 2$ looked at criminal activity, ${ }^{31,48}$ and 1 investigated risk behaviours for HIV infection. ${ }^{43}$ Additional details, including which variables each study controlled for, are provided in Table 1 . We attempted to obtain data necessary to calculate odds ratios when such data were not included in a study. We contacted 12 authors for missing data, but our requests were unmet. Additional details by outcome are presented in Supplemental Table S2, Appendix 1.

All the studies had a moderate or high risk of bias on at least 1 Newcastle-Ottawa Scale criterion. Eight studies did not adjust for any confounding variables. ${ }^{17,31,33-35,44,46,47}$ Complete details of the Newcastle-Ottawa Scale ratings are provided in Table 2 .

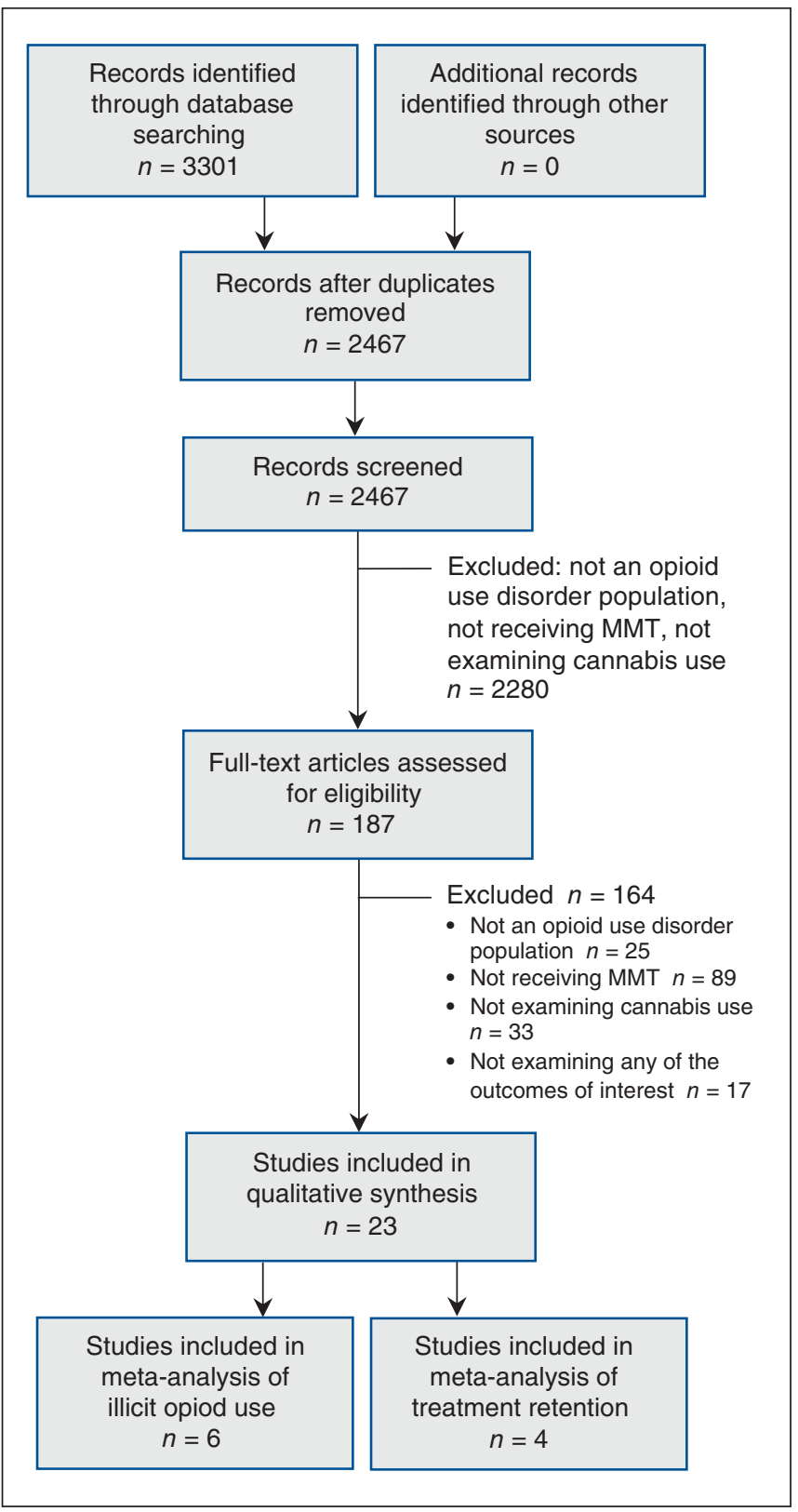

Figure 1: Flow diagram showing selection of included studies. Note: $\mathrm{MMT}=$ methadone maintenance treatment.

\section{Continued opioid use}

Twelve studies examined the relation between cannabis use and continued opioid use, with a total sample size of $3676 . .^{13,14,20,30-36,38,46}$ The majority of the studies showed no association between cannabis use and opioid use (Table 1).

There was significant heterogeneity among the studies. These results did not change when we excluded studies with a high risk of bias. Subgroup analyses by country and objective v. subjective measurement of cannabis did not reduce the heterogeneity. We present additional information on these studies in Supplemental Figures S3 and S4, Appendix 1.

The overall quality of evidence was very low, with critical issues of inconsistency and imprecision (Table 3), in addition to a moderate risk of bias. Owing to the nature of observational study designs, GRADE ratings of quality start at low, and any additional concerns in quality assessment make the quality very low.

\section{Treatment retention}

Eleven studies investigated cannabis use and retention in methadone maintenance therapy. ${ }^{13,17,31,32,34,39-43,46}$ Significant heterogeneity was seen $\left(I^{2}=90 \%\right)$. A sensitivity analysis conducted by excluding studies with high risk of bias to explain heterogeneity did not change the result. In the subgroup analysis by country, we found that studies conducted in the United States showed cannabis use to be significantly associated with decreased retention rates, whereas those conducted in Israel showed the opposite direction. Subgroup analyses by country had an $I^{2}$ of $0 \%$, indicating no heterogeneity. The overall quality of evidence was low, with quality issues related to inconsistency and imprecision. The funnel plot is presented in Supplemental Figure S2, Appendix 1.

\section{Secondary outcome measures}

Originally, we had aimed to further investigate the association between cannabis use and polydrug use, criminal activity, and risk behaviours for HIV infection and viral hepatitis. For polydrug use, we included 10 studies that investigated the association between cannabis use and use of cocaine, benzodiazepines, alcohol and various forms of cannabis. ${ }^{13,30,31,35,37,43-48}$ Owing to the various substances included and outcome measurements, we were unable to combine results for a metaanalysis. Two studies reporting on criminal activity could not be quantitatively analyzed..$^{31,48}$ One study reported on cannabis use and HIV infection. ${ }^{43}$

\section{Interpretation}

We included 23 studies that examined the association between cannabis use and opioid use and retention in methadone maintenance therapy. Meta-analysis of 6 of these studies showed high heterogeneity that affected the interpretation of the results. The overall quality of evidence was low, with high risk of bias. The results from individual studies suggest that cannabis use may potentially have no effect on opioid use in patients receiving methadone maintenance therapy. The results for treatment retention are inconclusive. We observed a difference 


\begin{tabular}{|c|c|c|c|c|c|c|c|}
\hline $\begin{array}{l}\text { Investigator, } \\
\text { year }\end{array}$ & Country & Study design & $\begin{array}{l}\text { Sample } \\
\text { size } \\
(\% \text { female })\end{array}$ & $\begin{array}{l}\text { Definition of } \\
\text { cannabis use }\end{array}$ & $\begin{array}{l}\text { Outcome(s) } \\
\text { of interest }\end{array}$ & Statistical analysis & Findings \\
\hline $\begin{array}{l}\text { Best et al.., } \\
1999\end{array}$ & UK & $\begin{array}{l}\text { Cross- } \\
\text { sectional }\end{array}$ & $200(30)$ & $\begin{array}{l}\text { Categorical: daily } \\
\text { users, occasional } \\
\text { users (not every } \\
\text { day in previous } \\
\text { month), nonusers }\end{array}$ & $\begin{array}{l}\text { Illicit opioid } \\
\text { use, } \\
\text { polydrug use }\end{array}$ & $\begin{array}{l}\text { ANOVA, post hoc } \\
\text { Scheffe test, linear } \\
\text { regression } \\
\text { Factors adjusted for: } \\
\text { use of various } \\
\text { substances, appetite, } \\
\text { overall health, } \\
\text { depression, anxiety }\end{array}$ & $\begin{array}{l}\text { Cannabis nonusers had more } \\
\text { occasions of heroin use than } \\
\text { occasional and daily users } \\
\text { Cannabis nonusers consumed } \\
\text { significantly more alcohol and } \\
\text { crack cocaine than occasional } \\
\text { and daily users }\end{array}$ \\
\hline $\begin{array}{l}\text { Epstein et } \\
\text { al., }{ }^{31} 2003\end{array}$ & US & $\begin{array}{l}\text { Secondary } \\
\text { analysis } \\
\text { ( } 3 \text { separate } \\
\text { analyses), } \\
12 \text { mo }\end{array}$ & $408(40.4)$ & $\begin{array}{l}\text { Dichotomized } \\
\text { cannabis use and } \\
\text { cannabis abuse/ } \\
\text { dependence } \\
\text { diagnosis }\end{array}$ & $\begin{array}{l}\text { Illicit opioid } \\
\text { use, } \\
\text { treatment } \\
\text { retention, } \\
\text { polydrug } \\
\text { use, criminal } \\
\text { activity }\end{array}$ & $\begin{array}{l}\text { Cox proportional } \\
\text { hazards regression } \\
\text { Factors adjusted for: } \\
\text { not stated } \\
\text { Confounders in the } \\
\text { regression }\end{array}$ & $\begin{array}{l}\text { No significant association } \\
\text { between cannabis use and } \\
\text { illicit opioid use } \\
\text { No significant association } \\
\text { between cannabis use and } \\
\text { treatment retention } \\
\text { Suggests an association } \\
\text { between cocaine abstinence } \\
\text { and cannabis use } \\
\text { Cannabis use category not } \\
\text { associated with any } \\
\text { differences in criminal activity }\end{array}$ \\
\hline $\begin{array}{l}\text { Levine et } \\
\text { al., }{ }^{32} 2015\end{array}$ & US & $\begin{array}{l}\text { Retrospective } \\
\text { cohort, } 1 \mathrm{yr}\end{array}$ & $290(40.3)$ & $\begin{array}{l}\text { Dichotomized } \\
\text { cannabis use }\end{array}$ & $\begin{array}{l}\text { Illicit opioid } \\
\text { use, } \\
\text { treatment } \\
\text { retention }\end{array}$ & $\begin{array}{l}\text { Logistic regression } \\
\text { Factors adjusted for: } \\
\text { total years of use of } \\
\text { various substances }\end{array}$ & $\begin{array}{l}\text { Not significant, but statistics } \\
\text { not reported } \\
\text { Cannabis-negative urine } \\
\text { associated with treatment } \\
\text { retention in both men and } \\
\text { women }\end{array}$ \\
\hline $\begin{array}{l}\text { Lions et } \\
\text { al.,.33 } 2014\end{array}$ & France & $\begin{array}{l}\text { Secondary } \\
\text { RCT analysis, } \\
45 \text { wk }\end{array}$ & $158(15.2)$ & $\begin{array}{l}\text { Dichotomized: } \\
\text { daily users v. } \\
\text { nondaily users }\end{array}$ & $\begin{array}{l}\text { Illicit opioid } \\
\text { use }\end{array}$ & $\begin{array}{l}\text { Univariate logistic } \\
\text { regression } \\
\text { Factors adjusted for: } \\
\text { not stated }\end{array}$ & $\begin{array}{l}\text { Pretreatment daily cannabis: } \\
\text { OR } 1.46 \text { ( } 95 \% \text { Cl 0.61-3.77), } \\
\text { NS } \\
\text { In-treatment daily cannabis: } \\
\text { OR } 2.81 \text { ( } 95 \% \text { Cl } 1.22-6.48)\end{array}$ \\
\hline $\begin{array}{l}\text { Nirenberg et } \\
\text { al., } 351996\end{array}$ & US & $\begin{array}{l}\text { Prospective } \\
\text { cohort, } 6 \text { mo }\end{array}$ & $70(1.4)$ & $\begin{array}{l}\text { Dichotomized } \\
\text { cannabis use and } \\
\text { categorical } \\
\text { ( } 4 \text { groups) }\end{array}$ & $\begin{array}{l}\text { Illicit opioid } \\
\text { use, } \\
\text { polydrug use }\end{array}$ & $\begin{array}{l}\text { ANOVA } \\
\text { Factors adjusted for: } \\
\text { not stated }\end{array}$ & $\begin{array}{l}\text { No significant association } \\
\text { between cannabis use and } \\
\text { illicit opioid use } \\
\text { No significant difference in use } \\
\text { of cocaine or benzodiazepine } \\
\text { between } 4 \text { cannabis groups }\end{array}$ \\
\hline $\begin{array}{l}\text { Proctor et } \\
\text { al., }{ }^{36} 2016 \dagger\end{array}$ & US & $\begin{array}{l}\text { Retrospective } \\
\text { cohort, } 12 \mathrm{mo}\end{array}$ & $2410(40.4)$ & $\begin{array}{l}\text { Dichotomized } \\
\text { cannabis use }\end{array}$ & $\begin{array}{l}\text { Illicit opioid } \\
\text { use }\end{array}$ & $\begin{array}{l}\text { Logistic regression } \\
\text { Factors adjusted for: } \\
\text { age, gender, } \\
\text { employment status, } \\
\text { ethnicity, marital } \\
\text { status, average daily } \\
\text { methadone dosage }\end{array}$ & $\begin{array}{l}\text { Intake cannabis values in } \\
\text { relation to opioid use at } 4 \text { time } \\
\text { points: } 3 \text { mo: OR } 1.17 \text { ( } 95 \% \mathrm{Cl} \\
0.83-1.63) ; 6 \text { mo: OR } 0.59 \\
(95 \% \mathrm{Cl} 0.32-1.10) ; 9 \text { mo: OR } \\
0.63(95 \% \mathrm{Cl} 0.24-1.66) ; \\
12 \mathrm{mo} \text { : OR } 0.23(95 \% \mathrm{Cl} \\
0.05-1.16)\end{array}$ \\
\hline
\end{tabular}

in the association between cannabis use and retention in methadone maintenance therapy in a subgroup analysis by country, specifically between studies conducted in the US and Israel. A previous study showed differences in retention in opioid use disorder treatment between Israel and the US, arguably due to various sociodemographic factors. ${ }^{41}$ However, given the conflicting findings by region, the overall effect of cannabis use on treatment retention in opioid use disorder is unknown.

The differences between our results and those of other investigators likely reflect how cannabis use was measured. 


\begin{tabular}{|c|c|c|c|c|c|c|c|}
\hline $\begin{array}{l}\text { Investigator, } \\
\text { year }\end{array}$ & Country & Study design & $\begin{array}{l}\text { Sample } \\
\text { size } \\
(\% \text { female })\end{array}$ & $\begin{array}{l}\text { Definition of } \\
\text { cannabis use* }\end{array}$ & $\begin{array}{l}\text { Outcome(s) } \\
\text { of interest }\end{array}$ & Statistical analysis & Findings \\
\hline $\begin{array}{l}\text { Saxon et } \\
\text { al., }{ }^{37} 1996\end{array}$ & US & $\begin{array}{l}\text { Prospective } \\
\text { cohort, } 18 \mathrm{mo}\end{array}$ & $353(38.2)$ & $\begin{array}{l}\text { Categorical: } \\
\text { 7-point scale } \\
\text { ranging from } 0 \\
\text { (never) to } 6 \\
\text { ( } \geq 4 \text { times per } \\
\text { day) }\end{array}$ & $\begin{array}{l}\text { Illicit opioid } \\
\text { use, } \\
\text { treatment } \\
\text { retention, } \\
\text { polydrug use }\end{array}$ & $\begin{array}{l}\text { Cox regression model } \\
\text { Factors adjusted for: } \\
\text { age, gender, previous } \\
\text { methadone treatment, } \\
\text { substance use }\end{array}$ & $\begin{array}{l}\text { No significant association } \\
\text { between cannabis use, and } \\
\text { illicit opioid use or treatment } \\
\text { retention } \\
\text { Significant association } \\
\text { between cannabis use and } \\
\text { cocaine use }\end{array}$ \\
\hline $\begin{array}{l}\text { Scavone et } \\
\text { al., }{ }^{13} 2013\end{array}$ & US & $\begin{array}{l}\text { Retrospective } \\
\text { cohort, } 9 \text { mo }\end{array}$ & $91(36.6)$ & $\begin{array}{l}\text { Dichotomized } \\
\text { cannabis use }\end{array}$ & $\begin{array}{l}\text { Illicit opioid } \\
\text { use, } \\
\text { treatment } \\
\text { retention, } \\
\text { polydrug use }\end{array}$ & $\begin{array}{l}\text { ANCOVA, parallel } \\
\text { ANCOVA } \\
\text { Factors adjusted for: } \\
\text { daily opioid } \\
\text { expenditure }\end{array}$ & $\begin{array}{l}\text { No significant relation } \\
\text { between frequency of } \\
\text { cannabis use in treatment and } \\
\text { opiate use } \\
\text { No significant association } \\
\text { between cannabis use and } \\
\text { treatment retention } \\
\text { Correlation between rates of } \\
\text { cannabis use and illicit } \\
\text { benzodiazepine use }\end{array}$ \\
\hline $\begin{array}{l}\text { Somers et } \\
\text { al., }{ }^{38} 2012\end{array}$ & Ireland & $\begin{array}{l}\text { Retrospective } \\
\text { cohort, } 15 \text { mo }\end{array}$ & 123 (NR) & $\begin{array}{l}\text { Dichotomized } \\
\text { cannabis use }\end{array}$ & $\begin{array}{l}\text { Illicit opioid } \\
\text { use }\end{array}$ & $\begin{array}{l}\text { Logistic regression } \\
\text { Factors adjusted for: } \\
\text { variables that were } \\
\text { significant from } \\
\text { univariate analysis, } \\
\text { did not explicitly state } \\
\text { which ones }\end{array}$ & $\begin{array}{l}\text { Baseline: OR 0.88 (95\% Cl } \\
0.67-1.15) ; 3 \text { mo: OR 0.79 } \\
\text { (95\% Cl 0.58-1.10); } 9 \text { mo: OR } \\
0.78 \text { (95\% Cl 0.55-1.20); } \\
15 \text { mo: OR } 1.45 \text { (95\% Cl } \\
0.82-2.50) ; \text { Total: adjusted OR } \\
0.32 \text { (95\% Cl 0.06-1.66) }\end{array}$ \\
\hline $\begin{array}{l}\text { Wasserman } \\
\text { et al.,20 } 1998\end{array}$ & US & $\begin{array}{l}\text { Prospective } \\
\text { cohort, } 6 \text { mo }\end{array}$ & $74(40.5)$ & $\begin{array}{l}\text { Dichotomized } \\
\text { cannabis use }\end{array}$ & $\begin{array}{l}\text { Illicit opioid } \\
\text { use }\end{array}$ & $\begin{array}{l}\text { Cox proportional } \\
\text { hazards regression } \\
\text { Factors adjusted for: } \\
\text { abstinence goals, } \\
\text { positive moods, } \\
\text { pleasant events, } \\
\text { negative moods, life } \\
\text { events, perceived } \\
\text { stress, opioid } \\
\text { withdrawal symptoms }\end{array}$ & $\begin{array}{l}\text { Significant association } \\
\text { between cannabis use and } \\
\text { illicit opioid use }\end{array}$ \\
\hline $\begin{array}{l}\text { Joe et al., }{ }^{39} \\
1998\end{array}$ & US & $\begin{array}{l}\text { Prospective } \\
\text { cohort, } 360 \mathrm{~d}\end{array}$ & 981 (39) & $\begin{array}{l}\text { Dichotomized: at } \\
\text { least weekly } \\
\text { marijuana use or } \\
\text { not }\end{array}$ & $\begin{array}{l}\text { Treatment } \\
\text { retention }\end{array}$ & $\begin{array}{l}\text { Hierarchical linear } \\
\text { regression model } \\
\text { Factors adjusted for: } \\
\text { age, ethnicity, marital } \\
\text { status, legal status, } \\
\text { employment status, } \\
\text { number of lifetime } \\
\text { arrests }\end{array}$ & $\begin{array}{l}\text { No significant association } \\
\text { between cannabis use and } \\
\text { treatment retention }\end{array}$ \\
\hline $\begin{array}{l}\text { Peles et } \\
\text { al. }{ }^{40} 2006\end{array}$ & Israel & $\begin{array}{l}\text { Prospective } \\
\text { cohort, } 11 \mathrm{yr}\end{array}$ & $492(27.2)$ & $\begin{array}{l}\text { Dichotomized } \\
\text { cannabis use }\end{array}$ & $\begin{array}{l}\text { Treatment } \\
\text { retention }\end{array}$ & $\begin{array}{l}\text { Fisher exact test, Cox } \\
\text { regression analysis } \\
\text { Factors adjusted for: } \\
\text { age, children, } \\
\text { methadone dosage, } \\
\text { use of various } \\
\text { substances }\end{array}$ & $\begin{array}{l}\text { No significant association } \\
\text { between cannabis use and } \\
\text { treatment retention }\end{array}$ \\
\hline $\begin{array}{l}\text { Peles et } \\
\text { al. }{ }^{41} 2008\end{array}$ & $\begin{array}{l}\text { US, } \\
\text { Israel }\end{array}$ & $\begin{array}{l}\text { Prospective } \\
\text { cohort, } 12 \text { mo }\end{array}$ & $794(31.0)$ & $\begin{array}{l}\text { Dichotomized } \\
\text { cannabis use }\end{array}$ & $\begin{array}{l}\text { Treatment } \\
\text { retention }\end{array}$ & $\begin{array}{l}\text { Kaplan-Meier survival } \\
\text { analysis with log rank } \\
\text { for cumulative retention, } \\
\text { Cox regression } \\
\text { Factors adjusted for: } \\
\text { methadone dosage, } \\
\text { age }\end{array}$ & $\begin{array}{l}\text { No significant association } \\
\text { between cannabis use and } \\
\text { treatment retention }\end{array}$ \\
\hline
\end{tabular}




\begin{tabular}{|c|c|c|c|c|c|c|c|}
\hline $\begin{array}{l}\text { Investigator, } \\
\text { year }\end{array}$ & Country & Study design & $\begin{array}{l}\text { Sample } \\
\text { size } \\
(\% \text { female) }\end{array}$ & $\begin{array}{c}\text { Definition of } \\
\text { cannabis use }\end{array}$ & $\begin{array}{l}\text { Outcome(s) } \\
\text { of interest }\end{array}$ & Statistical analysis & Findings \\
\hline $\begin{array}{l}\text { Schiff et } \\
\text { al.,42 } 2007\end{array}$ & Israel & $\begin{array}{l}\text { Retrospective } \\
\text { cohort, } 13 \mathrm{mo}\end{array}$ & $2683(14.1)$ & $\begin{array}{l}\text { Dichotomized } \\
\text { cannabis use }\end{array}$ & $\begin{array}{l}\text { Treatment } \\
\text { retention }\end{array}$ & $\begin{array}{l}\text { Logistic regression } \\
\text { Factors adjusted for: } \\
\text { age, gender }\end{array}$ & $\begin{array}{l}\text { Significant relation between } \\
\text { cannabis use and increased } \\
\text { treatment retention }\end{array}$ \\
\hline $\begin{array}{l}\text { Weizman et } \\
\text { al., }{ }^{43} 2004\end{array}$ & Israel & $\begin{array}{l}\text { Prospective } \\
\text { cohort, } 12 \text { mo }\end{array}$ & $283(\mathrm{NR})$ & $\begin{array}{l}\text { Dichotomized: } \\
\text { cannabis abuse } \\
\text { v. not }\end{array}$ & $\begin{array}{l}\text { Treatment } \\
\text { retention, } \\
\text { polydrug } \\
\text { use, risk } \\
\text { behaviours } \\
\text { for HIV } \\
\text { infection }\end{array}$ & $\begin{array}{l}\text { Cox regression } \\
\text { survival analysis } \\
\text { Factors adjusted for: } \\
\text { heroin, cocaine and } \\
\text { benzodiazepine } \\
\text { abuse }\end{array}$ & $\begin{array}{l}\text { No significant association } \\
\text { between cannabis use and } \\
\text { treatment retention } \\
\text { Significant association } \\
\text { between cannabis use and } \\
\text { use of benzodiazepine, } \\
\text { amphetamine and cocaine } \\
\text { Cannabis use was not related } \\
\text { to any risk behaviours } \\
\text { (statistics not reported) }\end{array}$ \\
\hline $\begin{array}{l}\text { White et al., } \\
2014^{17}\end{array}$ & US & $\begin{array}{l}\text { Retrospective } \\
\text { cohort, } \\
15-17 \text { mo }\end{array}$ & $604(39.4)$ & $\begin{array}{l}\text { Dichotomized } \\
\text { cannabis use }\end{array}$ & $\begin{array}{l}\text { Treatment } \\
\text { retention }\end{array}$ & $\begin{array}{l}\chi^{2} \text { test, Fisher exact } \\
\text { test } \\
\text { Factors adjusted for: } \\
\text { not stated }\end{array}$ & $\begin{array}{l}\text { At baseline, cannabis use was } \\
\text { significantly associated with } \\
\text { treatment retention }\end{array}$ \\
\hline $\begin{array}{l}\text { Bleich et } \\
\text { al., }{ }^{44} 1999\end{array}$ & Israel & $\begin{array}{l}\text { Prospective } \\
\text { cohort, } 12 \text { mo }\end{array}$ & $148(29.8)$ & $\begin{array}{l}\text { Positive result of } \\
\text { urine test for } \\
\text { cannabis during } \\
12 \text { th mo of } \\
\text { treatment‡ }\end{array}$ & Polydrug use & $\begin{array}{l}\chi^{2} \text { test } \\
\text { Factors adjusted for: } \\
\text { not stated }\end{array}$ & $\begin{array}{l}\text { Benzodiazepine abusers were } \\
\text { more likely to currently abuse } \\
\text { cannabis than nonabusers of } \\
\text { benzodiazepine }\end{array}$ \\
\hline $\begin{array}{l}\text { Peirce et } \\
\text { al., } 452009\end{array}$ & US & $\begin{array}{l}\text { Secondary } \\
\text { RCT analysis, } \\
12 \text { wk }\end{array}$ & $386(44)$ & $\begin{array}{l}\text { Cannabis use, } \\
\text { defined as } \\
\text { positive result of } \\
\text { testing of urine/ } \\
\text { breath sample } \\
\text { obtained at study } \\
\text { intake }\end{array}$ & Polydrug use & $\begin{array}{l}\text { Mixed-model } \\
\text { regression } \\
\text { Factors adjusted for: } \\
\text { age, gender, ethnicity, } \\
\text { employment status, } \\
\text { criminal activity, } \\
\text { additional } \\
\text { demographic factors }\end{array}$ & $\begin{array}{l}\text { Significant association } \\
\text { between cannabis use and } \\
\text { stimulant use }\end{array}$ \\
\hline $\begin{array}{l}\text { Saxon et } \\
\text { al., }{ }^{46} 1993\end{array}$ & US & $\begin{array}{l}\text { Cross- } \\
\text { sectional }\end{array}$ & $98(0)$ & $\begin{array}{l}\text { Dichotomized } \\
\text { cannabis use }\end{array}$ & Polydrug use & $\begin{array}{l}\text { Mann-Whitney test } \\
\text { Factors adjusted for: } \\
\text { not stated }\end{array}$ & $\begin{array}{l}\text { Significant association } \\
\text { between cannabis use and } \\
\text { other drug use }\end{array}$ \\
\hline $\begin{array}{l}\text { Strain et } \\
\text { al., } 471991\end{array}$ & US & $\begin{array}{l}\text { Cross- } \\
\text { sectional }\end{array}$ & $66(45)$ & $\begin{array}{l}\text { Dichotomized: } \\
\text { those with } \\
\text { v. without history } \\
\text { of cannabis use } \\
\text { diagnosis }\end{array}$ & Polydrug use & $\begin{array}{l}\text { Z-test } \\
\text { Factors adjusted for: } \\
\text { not stated }\end{array}$ & $\begin{array}{l}\text { No significant association } \\
\text { between cannabis use and } \\
\text { use of alcohol, sedatives and } \\
\text { cocaine }\end{array}$ \\
\hline $\begin{array}{l}\text { Bell et al., }{ }^{48} \\
1997\end{array}$ & Australia & $\begin{array}{l}\text { Prospective } \\
\text { cohort, } 12 \text { mo }\end{array}$ & $304(43.1)$ & $\begin{array}{l}\text { Continuous: } \\
\text { average daily use } \\
\text { of cannabis in } \\
\text { previous month }\end{array}$ & $\begin{array}{l}\text { Criminal } \\
\text { activity }\end{array}$ & $\begin{array}{l}\text { Multiple linear } \\
\text { regression } \\
\text { Factors adjusted for: } \\
\text { age, gender, } \\
\text { employment, } \\
\text { benzodiazepine use, } \\
\text { cost of drugs }\end{array}$ & $\begin{array}{l}\text { Cannabis use was significant } \\
\text { predictor of criminal activity at } \\
12 \mathrm{mo}\end{array}$ \\
\hline \multicolumn{8}{|c|}{$\begin{array}{l}\text { Note: } \text { ANCOVA = analysis of covariance, } \text { ANOVA = analysis of variance, } \mathrm{Cl}=\text { confidence interval, } \mathrm{NR}=\text { not reported, } \mathrm{NS}=\text { not significant, } \mathrm{OR}=\text { odds ratio, } \mathrm{RCT}= \\
\text { randomized controlled trial. } \\
\text { *"Dichotomized cannabis use" means users versus nonusers or at least } 1 \text { positive urine screen result versus none, unless specified otherwise. } \\
\text { †This study had too many results to present in this table, so we included only intake cannabis values in relation to opioid use at all time points. See study for more results. } \\
\ddagger \text { An abuser of any substance of abuse was defined as having a positive urine test result for that substance during the 12th month of treatment. }\end{array}$} \\
\hline
\end{tabular}

For instance, studies in our review dichotomized cannabis use in some way, categorizing people based on any cannabis use versus no use or grouping those who used more than a certain amount of cannabis versus those who used less. This choice of measurement was likely made because estimating the amount and type/strength/concentration of cannabis is challenging, ${ }^{49}$ and it makes the establishment of a dose-response relation in any of these studies impossible. ${ }^{50}$ This choice could obscure an association in either direction. Heavy use of cannabis has been reported to be associated with adverse health effects..$^{51,52}$ The studies in our systematic review did not distinguish between cannabis use disorder and recreational cannabis use. Compared to recreational users, patients with cannabis use disorder have high rates of comorbid psychiatric disorders, ${ }^{53}$ which are associated with poorer treatment outcomes. ${ }^{54}$ Peirce and colleagues ${ }^{45}$ suggested that a 


\begin{tabular}{|c|c|c|c|c|c|c|c|c|}
\hline Investigator & $\begin{array}{l}\text { Selection bias: } \\
\text { is source } \\
\text { population } \\
\text { representative? }\end{array}$ & \multicolumn{2}{|c|}{ Performance bias } & \multicolumn{2}{|c|}{ Detection bias } & \multicolumn{2}{|c|}{ Information bias } & $\begin{array}{l}\text { Total } \\
\text { score* }\end{array}$ \\
\hline Best et al. ${ }^{30}$ & 2 & 3 & 2 & 1 & 1 & 2 & 0 & 11 \\
\hline Bleich et al. ${ }^{44}$ & 1 & 1 & 0 & 1 & 1 & 1 & 3 & 8 \\
\hline $\begin{array}{l}\text { Epstein et } \\
\text { al. }^{31}\end{array}$ & 0 & 1 & 0 & 3 & 2 & 3 & 3 & 12 \\
\hline Joe et al. ${ }^{39}$ & 3 & 3 & 3 & 3 & 2 & 3 & 3 & 20 \\
\hline $\begin{array}{l}\text { Nirenberg et } \\
\text { al. }{ }^{35}\end{array}$ & 2 & 1 & 0 & 1 & 1 & 3 & 3 & 11 \\
\hline Peirce et al. ${ }^{45}$ & 1 & 3 & 3 & 3 & 3 & 3 & 3 & 19 \\
\hline Peles et al. ${ }^{40}$ & 3 & 3 & 3 & 3 & 3 & 3 & 3 & 21 \\
\hline Peles et al. ${ }^{41}$ & 3 & 3 & 3 & 3 & 3 & 3 & 3 & 21 \\
\hline Proctor et al. ${ }^{36}$ & 3 & 3 & 3 & 1 & 1 & 3 & 3 & 17 \\
\hline Saxon et al. ${ }^{46}$ & 1 & 1 & 0 & 1 & 1 & 2 & 3 & 9 \\
\hline Saxon et al. ${ }^{37}$ & 2 & 2 & 3 & 2 & 2 & 2 & 3 & 16 \\
\hline $\begin{array}{l}\text { Scavone et } \\
\text { al. } .^{13}\end{array}$ & 1 & 1 & 3 & 1 & 2 & 1 & 2 & 11 \\
\hline White et al. ${ }^{17}$ & 2 & 3 & 0 & 1 & 2 & 2 & 3 & 13 \\
\hline $\begin{array}{l}\text { Zielinski et } \\
\text { al. }^{14}\end{array}$ & 3 & 3 & 3 & 3 & 1 & 2 & 3 & 18 \\
\hline
\end{tabular}

diagnosis of cannabis use disorder in the previous 12 months is associated with less stimulant drug use during methadone maintenance therapy, whereas recent (in the previous month) cannabis use is associated with more stimulant use. Their study shows that the recency of cannabis use may affect the use of other drugs during methadone maintenance therapy. It is possible that patients in that study who used cannabis had more severe opioid-related problems or other factors driving their use, such as pain, which would have created a confounding effect; hence, the differences seen in the association of cannabis use with opioid use may have varied by the population of interest (e.g., pain or addiction cohorts).

Our findings suggest that cannabis use does not affect treatment outcomes for patients receiving methadone maintenance therapy. In these cases, it was not an exit drug. The broad negative health effects of heavy cannabis use have been well documented. ${ }^{51,52} \mathrm{We}$ should continue to counsel patients on the potential risks of cannabis use while emphasizing that we have no evidence to support the use of cannabis in opioid use disorder treatment. Previous studies showed that, in jurisdictions with medical cannabis laws, fewer prescription opioids were issued. ${ }^{21,22}$ Even in populations with pain conditions and not opioid addiction, those observations do not show that a reduction in opioid prescription was solely due to cannabis as a replacement for opioids. ${ }^{21,22}$ A national cohort study investigating cannabis use in patients prescribed opioids for pain showed that cannabis use did not reduce opioid use and was associated with worse pain control and psychiatric symptoms. ${ }^{55}$ 
Table 3: Grading of Recommendations Assessment, Development and Evaluation evidence profile for primary outcomes

\begin{tabular}{|c|c|c|c|c|c|c|c|c|}
\hline \multirow{2}{*}{$\begin{array}{l}\text { Outcome, } \\
\text { no. of } \\
\text { studies }\end{array}$} & \multicolumn{6}{|c|}{ Quality assessment } & \multirow[b]{2}{*}{ Quality } & \multirow[b]{2}{*}{ Importance } \\
\hline & Study design & $\begin{array}{c}\text { Risk of } \\
\text { bias }\end{array}$ & Inconsistency & Indirectness & Imprecision & $\begin{array}{c}\text { Other } \\
\text { considerations }\end{array}$ & & \\
\hline $\begin{array}{l}\text { Illicit opioid } \\
\text { use, } 7\end{array}$ & $\begin{array}{l}\text { Observational } \\
\text { studies }\end{array}$ & Serious* & $\begin{array}{l}\text { Very } \\
\text { serioust‡ }\end{array}$ & Not serious & $\begin{array}{l}\text { Very } \\
\text { serious§ }\end{array}$ & None & $\begin{array}{l}\oplus \mathrm{OOO} \\
\text { VERY LOW }\end{array}$ & CRITICAL \\
\hline Retention, 4 & $\begin{array}{l}\text { Observational } \\
\text { studies }\end{array}$ & $\begin{array}{l}\text { Not } \\
\text { serious }\end{array}$ & Serioust & Not serious & $\begin{array}{l}\text { Very } \\
\text { serious§ }\end{array}$ & None & $\begin{array}{l}\oplus \mathrm{OOO} \\
\text { VERY LOW }\end{array}$ & CRITICAL \\
\hline $\begin{array}{l}\text { *Moderate risk } \\
\text { †Point estimate } \\
\text { †Heterogeneity } \\
\text { §Small samples }\end{array}$ & $\begin{array}{l}\text { Is across studies. } \\
\text { y widely across st } \\
\text { explained by subg } \\
\text { le pooled } 95 \% \text { cor }\end{array}$ & $\begin{array}{l}\text { es, little over } \\
\text { p analyses. } \\
\text { ence interva }\end{array}$ & between individ & confidence inter & & & & \\
\hline
\end{tabular}

Further studies are needed to address the notion of cannabis use as a substitute for opioid use, whether for pain or for opioid use disorder. We need more research to understand the relations between opioid use, treatment outcomes and cannabis use in opioid use disorder. More investigation is needed to reconcile the findings of the prescription pattern studies with those of the patient population studies. The observational nature of the studies means that confounders such as the association of cannabis use with severity of opioid addiction or pain could obscure significant relations. Further research should include detailed definitions of cannabis use.

\section{Limitations}

There are limitations to be taken into consideration when interpreting our findings. The results come from small to medium-sized observational studies with limited data on confounding variables. For example, cannabis is seldom used alone; it is associated with polydrug use and with comorbid substance use disorders in patients receiving methadone maintenance therapy. ${ }^{43,56,57}$ Polydrug use and substance use disorders are associated with poorer outcomes in opioid use disorder. ${ }^{58}$ There were also methodological limitations. Our meta-analyses had substantial heterogeneity, partly because of methodological variability: there were differences in methadone maintenance therapy duration, associated psychosocial interventions, definition of cannabis use and outcome measures. Our subgroup analyses did not explain this heterogeneity for continued opioid use. The limited number of studies included in the meta-analyses precluded further subgroup analyses to identify other possible sources of heterogeneity, such as length of follow-up. Given the nature of observational studies, there are likely to be other sources of heterogeneity that cannot be detected because unknown confounding variables affect the outcomes. Finally, we were unable to do a complete grey literature search.

\section{Conclusion}

We found no consensus among studies that cannabis use is associated with reduced opioid use or longer treatment retention when used during methadone maintenance therapy in patients with opioid use disorder. The study limitations must be taken into account when interpreting these results. Further studies are needed to address and examine the notion of cannabis use and its effect on treatment outcomes in patients with opioid use disorder.

\section{References}

1. Hall AJ, Logan JE, Toblin RL, et al. Patterns of abuse among unintentional pharmaceutical overdose fatalities. FAMA 2008;300:2613-20.

2. Dunn KM, Saunders KW, Rutter CM, et al. Opioid prescriptions for chronic pain and overdose a cohort study. Ann Intern Med 2010;152:85-92.

3. Paulozzi LJ, Ryan GW. Opioid analgesics and rates of fatal drug poisoning in the United States. Am 7 Prev Med 2006;31:506-11.

4. Emergency department visits involving nonmedical use of selected prescription drugs, United States, 2004-2008. MMWR Morb Mortal Wkly Rep 2010;59: 705-9.

5. Kolodny A, Courtwright DT, Hwang CS, et al. The prescription opioid and heroin crisis: a public health approach to an epidemic of addiction. Annu Rev Public Health 2015;36:559-74.

6. Han B, Compton WM, Jones CM, et al. Nonmedical prescription opioid use and use disorders among adults aged 18 through 64 years in the United States, 2003-2013. 7AMA 2015;314:1468-78.

7. Connery HS. Medication-assisted treatment of opioid use disorder: review of the evidence and future directions. Harv Rev Psychiatry 2015;23:63-75.

8. Socías ME, Ahamad K. An urgent call to increase access to evidence-based opioid agonist therapy for prescription opioid use disorders. CMAJ 2016;188: 1208-9.

9. Farré M, Mas A, Torrens M, et al. Retention rate and illicit opioid use during methadone maintenance interventions: a meta-analysis. Drug Alcobol Depend 2002;65:283-90.

10. MacArthur GJ, Minozzi S, Martin N, et al. Opiate substitution treatment and HIV transmission in people who inject drugs: systematic review and metaanalysis. BM7 2012;345:e5945.

11. Bruneau J, Ahamad K, Goyer MÈ, et al. Management of opioid use disorders: a national clinical practice guideline. CMAf 2018;190:E247-57.

12. Shahid H, Bhatt M, Sanger N, et al. Association between family factors and illicit polysubstance use amongst methadone maintenance patients with opioid use disorder. Int 7 High Risk Behav Addict 2018;7:e58786.

13. Scavone JL, Sterling RC, Weinstein SP, et al. Impact of cannabis use during stabilization on methadone maintenance treatment. Am 7 Addict 2013;22: 344-51.

14. Zielinski L, Bhatt M, Sanger N, et al. Association between cannabis use and methadone maintenance treatment outcomes: an investigation into sex differences. Biol Sex Differ 2017;8:8.

15. Zielinski L, Bhatt M, Eisen RB, et al. Association between cannabis use and treatment outcomes in patients receiving methadone maintenance treatment: a systematic review protocol. Syst Rev 2016;5:139.

16. Timko C, Schultz NR, Cucciare MA, et al. Retention in medication-assisted treatment for opiate dependence: a systematic review. 7 Addict Dis 2016;35:22-35.

17. White WL, Campbell MD, Spencer RD, et al. Patterns of abstinence or continued drug use among methadone maintenance patients and their relation to treatment retention. 7 Psychoactive Drugs 2014;46:114-22.

18. Taylor OD. Poly substance use in methadone maintenance therapy (MMT) patients. 7 Hum Behav Soc Environ 2015;25:822-9.

19. Vowles KE, McEnteea ML, Julnes TF, et al. Rates of opioid misuse, abuse, and addiction in chronic pain: a systematic review and data synthesis. Pain 2015;156:569-76.

20. Wasserman DA, Weinstein MG, Havassy BE, et al. Factors associated with lapses to heroin use during methadone maintenance. Drug Alcobol Depend 1998; 52:183-92. 
21. Bradford AC, Bradford WD, Abraham A, et al. Association between US state medical cannabis laws and opioid prescribing in the Medicare part D population. 7AMA Intern Med 2018;178:667-72.

22. Bachhuber MA, Saloner B, Cunningham CO, et al. Medical cannabis laws and opioid analgesic overdose mortality in the United States, 1999-2010. 7AMA Intern Med 2014;174:1668-73.

23. Angell T. V.A. issues new medical marijuana policy for military veterans. Forbes 2017 Dec. 19. Available: https://www.forbes.com/sites/tomangell/2017/12/19/ $\mathrm{v}$-a-issues-new-medical-marijuana-policy-for-military-veterans/\#6d1ff4215b90 (accessed 2019 Oct. 29).

24. Moher D, Liberati A, Tetzlaff J, et al. Preferred reporting items for systematic reviews and meta-analyses: the PRISMA statement. PLoS Med 2009;6: e1000097.

25. Bawor M, Dennis BB, Anglin R, et al. Sex differences in outcomes of methadone maintenance treatment for opioid addiction: a systematic review protocol. Syst Rev 2014;3:45.

26. Guyatt GH, Oxman AD, Schünemann HJ, et al. GRADE guidelines: a new series of articles in the Journal of Clinical Epidemiology. 7 Clin Epidemiol 2011; 64:380-2.

27. Higgins J, Green S, editors. Cochrane handbook for systematic reviews of interventions. Version 5.1.0 [updated March 2011]. Oxford (UK): Cochrane Collaboration; 2011.

28. Stang A. Critical evaluation of the Newcastle-Ottawa Scale for the assessment of the quality of nonrandomized studies in meta-analyses. Eur 7 Epidemiol 2010;25:603-5.

29. Lau J, Ioannidis JPA, Terrin N, et al. Evidence based medicine: the case of the misleading funnel plot. BM7 2006;333:597.

30. Best D, Gossop M, Greenwood J, et al. Cannabis use in relation to illicit drug use and health problems among opiate misusers in treatment. Drug Alcohol Rev 1999;18:31-8.

31. Epstein DH, Preston KL. Does cannabis use predict poor outcome for heroindependent patients on maintenance treatment? Past findings and more evidence against. Addiction 2003;98:269-79.

32. Levine AR, Lundahl LH, Ledgerwood DM, et al. Gender-specific predictors of retention and opioid abstinence during methadone maintenance treatment. f Subst Abuse Treat 2015;54:37-43.

33. Lions C, Carrieri MP, Michel L, et al. Predictors of non-prescribed opioid use after one year of methadone treatment: an attributable-risk approach (ANRSMethaville trial). Drug Alcohol Depend 2014;135:1-8.

34. Nava F, Manzato E, Lucchini A. Chronic cannabis use does not affect the normalization of hypothalamic-pituitary-adrenal (HPA) axis induced by methadone in heroin addicts. Prog Neuropsychopharmacol Biol Psychiatry 2007; 31:1089-94.

35. Nirenberg TD, Cellucci T, Liepman MR, et al. Cannabis versus other illicit drug use among methadone maintenance patients. Psychol Addict Behav 1996; $10: 222-7$.

36. Proctor SL, Copeland AL, Kopak AM, et al. Outcome predictors for patients receiving methadone maintenance treatment: findings from a retrospective multi-site study. 7 Subst Use 2016;21:601-13.

37. Saxon AJ, Wells EA, Fleming C, et al. Pre-treatment characteristics, program philosophy and level of ancillary services as predictors of methadone maintenance treatment outcome. Addiction 1996;91:1197-209.

38. Somers CJ, O'Connor J. Retrospective study of outcomes, for patients admitted to a drug treatment centre board. Ir Med 7 2012;105:295-8.

39. Joe GW, Simpson DD, Broome KM. Effects of readiness for drug abuse treatment on client retention and assessment of process. Addiction 1998;93:1177-90.

40. Peles E, Schreiber S, Adelson M. Factors predicting retention in treatment: 10 -year experience of a methadone maintenance treatment (MMT) clinic in Israel. Drug Alcohol Depend 2006;82:211-7.

41. Peles E, Linzy S, Kreek MJ, et al. One-year and cumulative retention as predictors of success in methadone maintenance treatment: a comparison of two clinics in the United States and Israel. 7 Addict Dis 2008;27:11-25.

42. Schiff M, Levit S, Moreno RC. Retention and illicit drug use among methadone patients in Israel: a gender comparison. Addict Behav 2007;32:2108-19.

43. Weizman T, Gelkopf M, Melamed Y, et al. Cannabis abuse is not a risk factor for treatment outcome in methadone maintenance treatment: a 1-year prospective study in an Israeli clinic. Aust N Z 7 Psychiatry 2004;38:42-6.

44. Bleich AVI, Gelkopf M, Schmidt V, et al. Correlates of benzodiazepine abuse in methadone maintenance treatment: a 1-year prospective study in an Israeli clinic. Addiction 1999;94:1533-40.
45. Peirce JM, Petry NM, Roll JM, et al. Correlates of stimulant treatment outcome across treatment modalities. Am 7 Drug Alcobol Abuse 2009;35:48-53.

46. Saxon AJ, Calsyn DA, Greenberg D, et al. Urine screening for marijuana among methadone-maintained patients. Am 7 Addict 1993;2:207-11.

47. Strain EC, Brooner RK, Bigelow GE. Clustering of multiple substance use and psychiatric diagnoses in opiate addicts. Drug Alcobol Depend 1991;27:127-34

48. Bell J, Mattick R, Hay A, et al. Methadone maintenance and drug-related crime. 7 Subst Abuse 1997;9:15-25.

49. van der Pol P, Liebregts N, de Graaf R, et al. Validation of self-reported cannabis dose and potency: an ecological study. Addiction 2013;108:1801-8.

50. Scavone JL, Sterling RC, Van Bockstaele EJ. Cannabinoid and opioid interactions: implications for opiate dependence and withdrawal. Neuroscience 2013 248:637-54

51. Volkow ND, Baler RD, Compton WM, et al. Adverse health effects of marijuana use. $N$ Engl 7 Med 2014;370:2219-27.

52. Volkow ND, Swanson JM, Evins AE, et al. Effects of cannabis use on human behavior, including cognition, motivation, and psychosis: a review. $7 A M A$ Psychiatry 2016;73:292-7.

53. Khan SS, Secades-Villa R, Okuda M, et al. Gender differences in cannabis use disorders: results from the national epidemiologic survey of alcohol and related conditions. Drug Alcohol Depend 2013;130:101-8.

54. Rosic T, Naji L, Bawor M, et al. The impact of comorbid psychiatric disorders on methadone maintenance treatment in opioid use disorder: a prospective cohort study. Neuropsychiatr Dis Treat 2017;13:1399-408.

55. Campbell G, Hall WD, Peacock A, et al. Effect of cannabis use in people with chronic non-cancer pain prescribed opioids: findings from a 4-year prospective cohort study. Lancet Public Health 2018;3:e341-50.

56. Degenhardt L, Hall W, Lynskey $M$. The relationship between cannabis use and other substance use in the general population. Drug Alcohol Depend 2001; 64:319-27.

57. Blanco C, Hasin DS, Wall MM, et al. Cannabis use and risk of psychiatric disorders: prospective evidence from a US national longitudinal study. $7 A M A$ Psychiatry 2016;73:388-95.

58. Kelly SM, O'Grady KE, Mitchell SG, et al. Predictors of methadone treatment retention from a multi-site study: a survival analysis. Drug Alcobol Depend 2011; $117: 170-5$.

Affiliations: Statistical Sciences Program (McBrien), University of Toronto, Toronto, Ont.; Michael G. DeGroote School of Medicine (Luo), Medical Sciences Graduate Program (Sanger), Neuroscience Graduate Program (Zielinski) and Health Research Methodology Graduate Program (Bhatt), McMaster University, Hamilton, Ont.; St. George's Hospital Medical School (Zhu), University of London, London, UK; Northern Ontario School of Medicine (Marsh), Sudbury, Ont.; Biostatistics Unit (Thabane), Research Institute at St. Joes, St. Joseph's Healthcare Hamilton; Departments of Health Research Methods, Evidence and Impact (Thabane, Samaan) and Psychiatry and Behavioural Neurosciences (Samaan), McMaster University, Hamilton, Ont.

Contributors: Heather McBrien and Zainab Samaan contributed to the study conception. Heather McBrien developed the search strategy. Heather McBrien, Candice Luo and Zainab Samaan analyzed and interpreted the data. Heather McBrien, Candice Luo, Nitika Sanger, Laura Zielinski, Meha Bhatt, Xi Ming Zhu, David Marsh and Lehana Thabane contributed to drafting the manuscript. Candice Luo, Nitika Sanger, Laura Zielinski, Meha Bhatt, Xi Ming Zhu, David Marsh, Lehana Thabane and Zainab Samaan critically revised the manuscript for important intellectual content. All of the authors contributed to the study design, approved the final version to be published and agreed to act as guarantors of the work.

Funding: This work was funded by Catalyst Grant 155404 from the Canadian Institutes of Health Research.

Supplemental information: For reviewer comments and the original submission of this manuscript, please see www.cmajopen.ca/content/7/4/ E665/suppl/DC1. 